\title{
Clinical presentation of neuroendocrine cancer metastasis to an anterior clinoid process meningioma invading superior orbital fissure. A case report
}

\begin{abstract}
Anna Rzehak ${ }^{1}$, Michat Roman Sobstyl ${ }^{1}$, Teresa Wierzba-Bobrowicz ${ }^{2}$, Piotr Bojarski ${ }^{1}$, Wiesława Grajkowska ${ }^{3}$
${ }^{1}$ Department of Neurosurgery, Institute of Psychiatry and Neurology, Warsaw, Poland, ${ }^{2}$ Department of Neuropathology, Institute of Psychiatry and Neurology, Warsaw, Poland, ${ }^{3}$ Department of Pathomorphology, Centre of Postgraduate Medical Education, Warsaw, Poland
\end{abstract}

\begin{abstract}
Metastasis to a meningioma is an uncommon phenomenon however reported in the literature. Meningiomas are common primary intracranial tumours which most frequently occur to be a recepient of metastases. A 66-yearold female presented with rapid development of visual acuity and visual field loss in the right eye with ipsilateral oculomotor nerve palsy. Magnetic resonance imaging (MRI) showed well-defined tumour intensely enhanced with contrast like a typical skull base meningioma. The neuropathological examination revealed two different morphological fragments of the tumour. In the cell-rich part of the tumour, immunopositivity for $C K$, chromogranin, and $S Y$ were detected. The less cellular portion of the tumour, immunopositivity to epithelial membrane antigen (EMA) and vimentin were detected. To our knowledge, we present the first rare metastasis of neuroendocrine carcinoma to the medial sphenoid meningioma that preceded the clinical symptoms of systemic neuroendocrine carcinoma.
\end{abstract}

Key words: neuroendocrine cancer, meningioma, brain metastasis, tumour-to-meningioma metastasis.

\section{Introduction}

Tumour-to-meningioma metastasis (TTMM) is a rare phenomenon however in last few years repeatedly reported in the literature $[12,22]$. Meningiomas most commonly occur to be a target by metastatic spread of cancers. In most of the known cases the origin of TTMM was breast and lung carcinomas although there have been also reported cases of kidney, prostate and gastrointestinal tract carcinomas as well as lymphomas and melanomas as donor tumours [22].
Meningiomas are common primary intracranial tumours. Vast majority of meningiomas are slow-growing benign lesions $[9,24]$. They usually occur as solitary, encapsulated tumours arising from meninges with strong contrast enhancement and blood supply derived most commonly from external carotid artery branches or meningeal branches of the internal carotid artery. However, meningiomas can also appear as en plaque forms or intraventricular. The typical radiological features of meningiomas found on an unenhanced computed tomography (CT) include hyperdense lobular masses which might be 
to some extent calcified with homogenous enhancement after intravenous contrast agent application. On a magnetic resonance imaging (MRI) meningiomas typically appear as round, circumscribed tumours witch attachment to the dura matter. They usually present as hypointense to isointense on T1 weighted imaging and iso- to hyperintense on T2 weighted imaging. The dura mater thickening on the tumour circumference called as dura tail appear on post-contrast T1 weighted imaging and it is typical for benign meningiomas [10]. Histologically the pathognomonic features include whorls - sphere-forming meningothelial cells which at the last stage are transformed into psammoma bodies. The most common immunohistochemical (IHC) marker associated with meningioma is an epithelial membrane antigen (EMA) [9].

Sphenoid wing meningiomas are the third most common group of intracranial meningiomas. Varying locations of sphenoid wing meningiomas as well as clinical and anatomic features divide them to medial, middle and lateral sphenoid wing meningiomas [7]. Medial sphenoid wing meningiomas are attached over an anterior clinoid process, which indicates specific clinical presentation [23]. Visual field and visual acuity loss, visual blur and oculomotor palsy are most common symptoms [18].

In this article we report a rare case of neuroendocrine cancer metastasis to a sphenoid wing meningioma invading inferior orbital fissure.

\section{Case description}

A 66-year-old female who presented with rapidly evolving visual acuity, ptosis and diplopia was admitted to the Department of Neurosurgery. Symptoms as diplopia, visual field loss and eyelid droop in the right eye appeared 6 weeks prior to admission to hospital.
A neurological examination revealed visual acuity impairment, concentric visual field loss, ptosis, mydriasis and impairment of eye movements due to severe oculomotor nerve palsy on the right side. No other cranial nerves or neurological deficits were found. MRI of the brain was performed approximately 3 weeks after onset of symptoms. The MRI scan disclosed a contrast enhancing tumour invading the right optic canal and right superior orbital fissure. Blood test results showed mild normocytic anaemia with no further significant abnormalities and chest radiograph findings included unclear opacity in the lower lung field of the right lung.

Total resection was performed via right pterional craniotomy with anterior clinoidectomy, unroofing of the optic canal and opening of the optic nerve sheath (Simpson I). Post-operative CT of the brain showed normal postoperative imaging findings accurate to the surgical technique performed.

\section{Neuropathological findings}

For neuropathological examination tumour tissues were fixed in $4 \%$ buffered formaldehyde and embedded in paraffin and HE stained for the routine diagnostic procedures. The immunostaining was performed using antibodies to CK/clone AE1/AE3 (DAKO-M3515, 1 : 50), Ki67 (Thermo Fisher-MA5-14520, 1 : 75), EMA (Leica-NCL-L-EMA, 1 : 200), vimentin (LeicaNCL-L-VIM-572, 1 : 400), synaptophysin (Leica-NCLL-Synap-299, 1 : 150), chromogranin (DAKO-M0869, 1 : 100). Two different morphological fields were revealed in microscopic examination: cell-rich and cell-poor with intranuclear areas surrounded by marginated chromatin (Fig. 1A). The predominant component was positive for cytokeratin CKAE1/3, chromogranin, synaptophysin and the Ki67 proliferation index was greater than $50 \%$ (Figs. 1B, 2A, B, 3B). The cell-
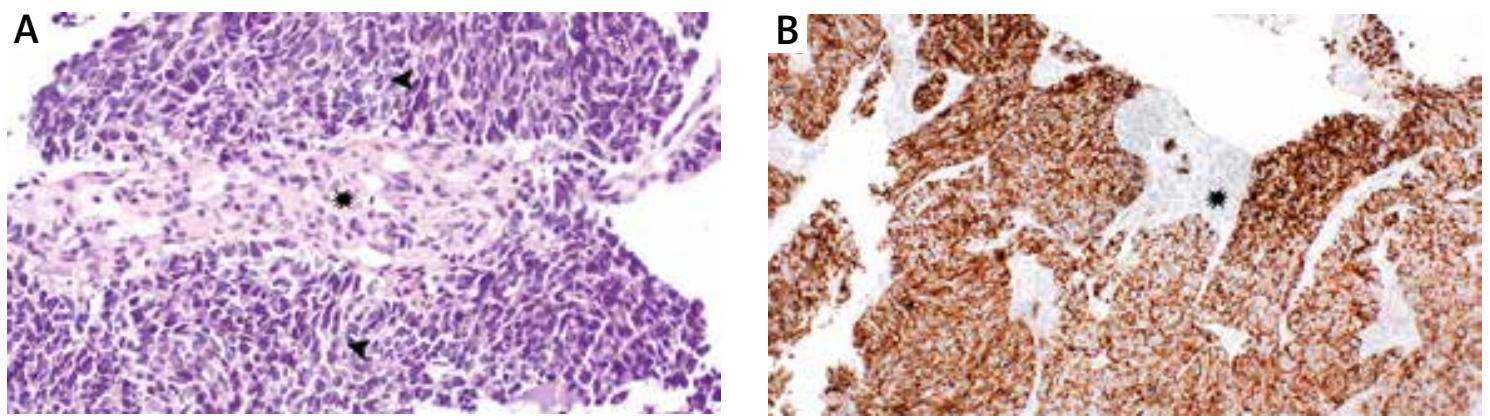

Fig. 1. Histopathology of the tumours. A) Metastasis of neuroendocrine carcinoma $(\wedge)$ and meningioma cells with intranuclear inclusions surrounded by marginated chromatin $\left({ }^{\star}\right), \mathrm{HE}$. B) Neuroendocrine carcinoma metastasis immunopositive $(\wedge)$ and meningioma negative reaction for CK AE $1 / 3\left({ }^{*}\right)$. 

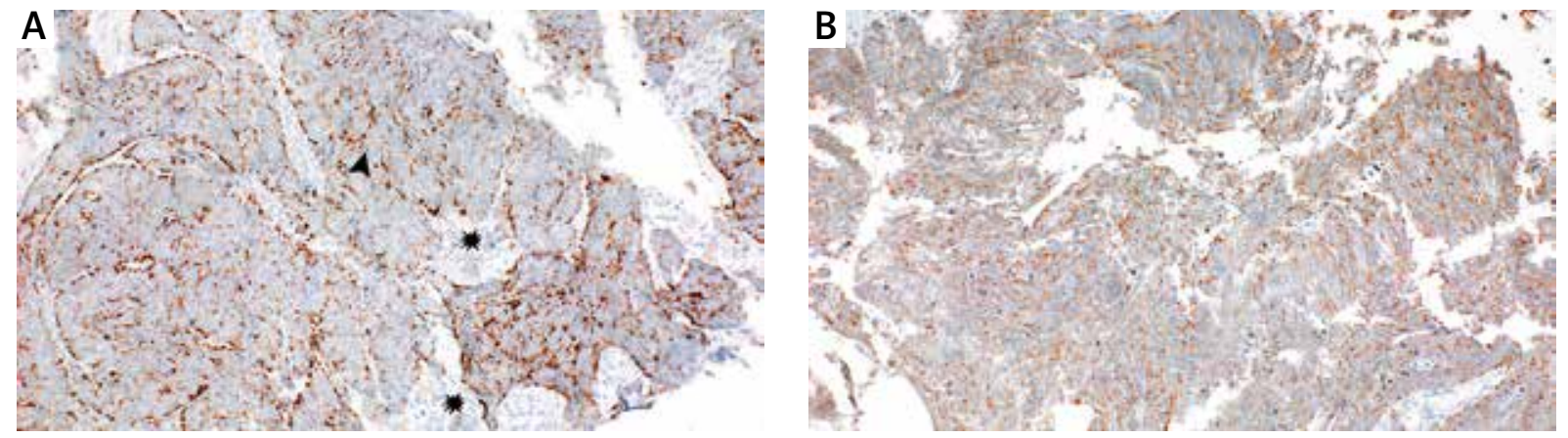

Fig. 2. Neuroendocrine carcinoma metastasis $\left({ }^{\wedge}\right)$. A) Chromogranin reaction, meningioma $\left({ }^{\star}\right)$. B) Synaptophysin reaction.
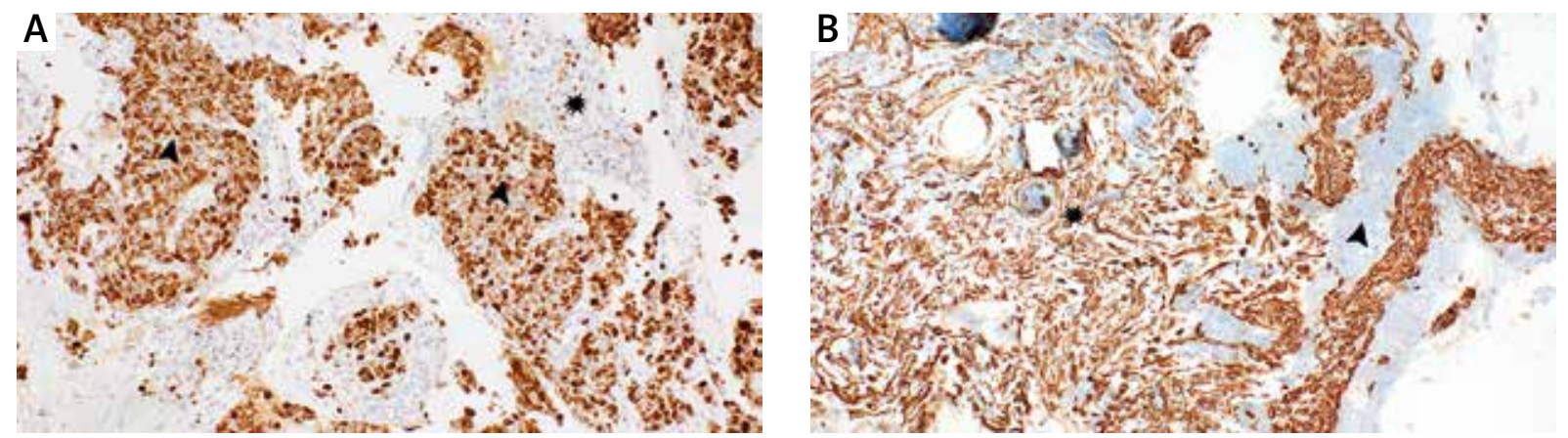

Fig. 3. Neuroendocrine carcinoma metastasis $\left({ }^{\wedge}\right)$, meningioma $\left({ }^{\star}\right)$. A) Ki67 expression. B) Vimentin expression.

poor fragment was vimentin positive with a proliferation index of about 1-2\% (Fig. 3A, B). In both tissue fragments immunopositivity for epithelial membrane (EMA) was detected. On this account neuroendocrine cancer metastasis to a meningioma was a final diagnosis.

Nearly two weeks after the surgery, the patient deteriorated due to pneumonia. She was admitted to the regional Internal Medicine Ward and also a positive SARS-CoV-2 test was detected. Chest and abdominal CT scans revealed multitudinous metastatic lesions in lungs, liver and peritoneum. Two weeks later the patient died due to advanced malignancies.

\section{Discussion}

Tumour-to-meningioma metastasis cases have been more frequently reported in last few years. According to Turner et al., in over one-third of reported cases metastases to a meningioma were the primary findings of previously undetected cancers. Lung and breast carcinoma appear as the origin of metastases in numerous cases $[6,16,22]$. To our knowledge, only two cases of neuroendocrine neo- plasm metastasis to a meningioma were reported in the literature $[3,20]$; in both cases clinical symptoms of systemic cancer appeared previously to TTMM occurrence. As far as we know, this is the first case report of TTMM as a primary manifestation of neuroendocrine cancer. In the present case, there are similarities between patient characteristics and meningiomas in the literature. Age of 66 years is comparable to average age reported in vast majority of meningiomas as well as female preponderance $[5,8,9]$. Furthermore, clinical presentation as progressive loss of visual sharpness and visual field are common symptoms of medial sphenoid wing meningiomas but they generally occur later in larger tumours than in the presented case. Although rapid development of symptoms differentiate meningioma from TTMM.

Neuroendocrine tumours (NETs) are a heterogenic group of cancer subtypes and fairly rare tumours constituting about $2 \%$ of malignancies [11,17,19,21]. Typically primary sites of NETs are the gastrointestinal tract and the lung [1]. The vast majority of NETs occur sporadically with female preponderance however some hereditary syndromes such as multiple endocrine neoplasia type 1 syndrome (MEN-1), multiple endocrine neoplasia type 2 syndrome (MEN-2), 
von Hippel-Lindau syndrome (VHL), neurofibromatosis and tuberous sclerosis are associated with NETs occurrence [14]. IHC findings include characteristic neuroendocrine markers such as CgA and Syn. Metastases to a brain occur approximately in $10 \%$ of reported NET cases and most frequently originating from bronchial NETs [2].

Several pathophysiological theories propose potential mechanisms of TTMM. Certain characteristics of meningiomas such as slow growth, indolent nature, low metabolic activity and rich vascularity permit the metastatic spread. Also, cell adhesion molecules particularly E-cadherin are correlated to TTMM occurrence. Unfortunately meningioma parameters, location of newly diagnosed meningioma, radiological imaging such as CT or MRI scans and also cancer origin or cancer status cannot distinguish meningiomas from TTMM. Turner et al. suggest that TTMM should be considered as possible diagnosis in patients in their 60 s to 80 s with newly diagnosed meningioma especially when it is symptomatic. There are also suggestions that magnetic resonance spectroscopy and perfusion MRI can serve in diagnosis prior to histological examination $[4,13]$.

\section{Conclusions}

Tumour-to-meningioma metastasis is still a rare incident. However, with increasing numbers of cases reported in the literature the necessity of providing better portraying of diagnosis and prognosis of TTMM is highly required for better understanding of this phenomenon.

\section{Disclosure}

The authors report no conflict of interest.

\section{Acknowledgements}

This work is supported by the "Digital Brain digital collection of the Institute Psychiatry and Neurology" (Project No. POPC.02.03.01-00.0042/18-00). The project "Digital Brain" is co-funded by the European Union and the Polish budget. The authors are very grateful to the First Polish Brain Bank in Institute Psychiatry and Neurology, Warsaw Poland.
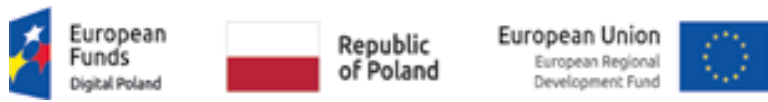

\section{References}

1. Ahmed M. Gastrointestinal neuroendocrine tumors in 2020. World J Gastrointest Oncol 2020; 12: 791-807.

2. Akimoto J, Fukuhara H, Suda T, Nagai K, Ichikawa M, Fukami S, Kohno M, Matsubayashi J, Nagao T. Clinicopathological analysis in patients with neuroendocrine tumors that metastasized to the brain. BMC Cancer 2016; 16: 36.

3. Bhojwani N, Huang J, Gupta A, Badve C, Cohen ML, Wolansky LJ. Rectal carcinoid tumor metastasis to a skull base meningioma. Neuroradiol J 2016; 29: 49-51.

4. Bulakbasi N, Kocaoglu M, Ors F, Tayfun C, Uçöz T. Combination of single-voxel proton MR spectroscopy and apparent diffusion coefficient calculation in the evaluation of common brain tumors. AJNR Am J Neuroradiol 2003; 24: 225-233.

5. Buerki RA, Horbinski CM, Kruser T, Horowitz PM, James CD, Lukas RV. An overview of meningiomas. Future Oncol 2018; 14: 2161-2177.

6. Chatani M, Nakagawa I, Yamada S, Sugimoto T, Hironaka Y, Nakamura M, Nakase H. Intracranial meningioma as initial clinical manifestation of occult lung carcinoma: case report. Neurol Med Chir (Tokyo) 2014; 54: 670-672.

7. Cushing H, Eisenhardt L. Meningiomas: their classification, regional behavior, life history, and surgical end results. Charles C Thomas, Springfield, Illinois 1938; 298-319.

8. Dolecek TA, Dressler EV, Thakkar JP, Liu M, Al-Qaisi A, Villano JL. Epidemiology of meningiomas post-Public Law 107-206: The Benign Brain Tumor Cancer Registries Amendment Act. Cancer 2015; 121: 2400-2410.

9. Goldbrunner R, Minniti G, Preusser M, Jenkinson MD, Sallabanda K, Houdart E, von Deimling A, Stavrinou P, Lefranc F, LundJohansen M, Moyal EC, Brandsma D, Henriksson R, Soffietti R, Weller M. EANO guidelines for the diagnosis and treatment of meningiomas. Lancet Oncol 2016; 17: 383-391.

10. Huang RY, Bi WL, Griffith B, Kaufmann TJ, la Fougère C, Schmidt NO, Tonn JC, Vogelbaum MA, Wen PY, Aldape K, Nassiri F, Zadeh G, Dunn IF, International Consortium on Meningiomas. Imaging and diagnostic advances for intracranial meningiomas. Neuro Oncol 2019; 21: 44-61.

11. Hung YP. Neuroendocrine tumors of the lung: updates and diagnostic pitfalls. Surg Pathol Clin 2019; 12: 1055-1071.

12. Iżycka-Świeszewska E, Czapiewski P, Szurowska E, Borof D, Wesołowski W, Kloc W. Cancer metastases to meningioma: apropos two cases. Folia Neuropathol 2013; 51: 340-346.

13. Jun P, Garcia J, Tihan T, McDermott MW, Cha S. Perfusion MR imaging of an intracranial collision tumor confirmed by image-guided biopsy. AJNR Am J Neuroradiol 2006; 27: 94-97.

14. Lewis MA. Hereditary syndromes in neuroendocrine tumors. Curr Treat Options Oncol 2020; 21: 50.

15. Masaya-anon P, Lorpattanakasem J. Intracranial tumors affecting visual system: 5-year review in Prasat Neurological Institute. J Med Assoc Thai 2008; 91: 515-519.

16. Neville IS, Solla DF, Oliveira AM, Casarolli C, Teixeira MJ, Paiva WS. Suspected tumor-to-meningioma metastasis: A case report. Oncol Lett 2017; 13: 1529-1534. 
17. Patel N, Benipal B. Incidence of neuroendocrine tumors in the United States from 2001-2015: a United States cancer statistics analysis of 50 states. Cureus 2019; 11: 4322.

18. Samal S, Patnaik A, Sahu FM, Purkait S. Altered expression of epigenetic modifiers EZH2, H3K27me3, and DNA methyltransferases in meningiomas - prognostic biomarkers for routine practice. Folia Neuropathol 2020; 58: 133-142.

19. Singh S, Bergsland EK, Card CM, Hope TA, Kunz PL, Laidley DT, Lawrence B, Leyden S, Metz DC, Michael M, Modahl LE, Myrehaug S, Padda SK, Pommier RF, Ramirez RA, Soulen M, Strosberg J, Sung A, Thawer A, Wei B, Xu B, Segelov E. Commonwealth Neuroendocrine Tumour Research Collaboration and the North American Neuroendocrine Tumor Society Guidelines for the Diagnosis and Management of Patients With Lung Neuroendocrine Tumors: An International Collaborative Endorsement and Update of the 2015 European Neuroendocrine Tumor Society Expert Consensus Guidelines. J Thorac Oncol 2020; 15 1577-1598.

20. Smith TW, Wang SY, Schoene WC. Malignant carcinoid tumor metastatic to a meningioma. Cancer 1981; 47: 1872-1877.

21. Trikalinos NA, Tan BR, Amin M, Liu J, Govindan R, Morgensztern D. Effect of metastatic site on survival in patients with neuroendocrine neoplasms (NENs). An analysis of SEER data from 2010 to 2014. BMC Endocr Disord 2020; 20: 44.

22. Turner N, Kaye AH, Paldor I. Metastases to meningioma-review and meta-analysis. Acta Neurochir (Wien) 2021; 163: 699-709.

23. Verma SK, Sinha S, Sawarkar DP, Singh PK, Gupta D, Agarwal D, Satyarthee G, Kumar R, Singh M, Suri A, Chandra PS, Kale SS, Sharma BS. Medial sphenoid wing meningiomas: Experience with microsurgical resection over 5 years and a review of literature. Neurol India 2016; 64: 465-475.

24. Wiemels J, Wrensch M, Claus EB. Epidemiology and etiology of meningioma. J Neurooncol 2010; 99: 307-314.

25. Wu Q, Zheng J, Dou C, Qi S. Forkhead Box M1 promotes the growth and tube formation of human malignant meningioma cells via the aryl hydrocarbon receptor signaling pathway. Folia Neuropathol 2020; 58: 223-236. 